\title{
Identification and characterization of four Drosophila suzukii cellularization genes and their promoters
}

Ying Yan $^{1,2^{*}}$, Syeda A. Jaffri ${ }^{1}$, Jonas Schwirz ${ }^{2}$, Carl Stein ${ }^{1}$ and Marc F. Schetelig ${ }^{1,2^{*}}$

\begin{abstract}
Background: The spotted-wing Drosophila (Drosophila suzukii) is a widespread invasive pest that causes severe economic damage to fruit crops. The early development of D. suzukii is similar to that of other Drosophilids, but the roles of individual genes must be confirmed experimentally. Cellularization genes coordinate the onset of cell division as soon as the invagination of membranes starts around the nuclei in the syncytial blastoderm. The promoters of these genes have been used in genetic pest-control systems to express transgenes that confer embryonic lethality. Such systems could be helpful in sterile insect technique applications to ensure that sterility (bi-sex embryonic lethality) or sexing (female-specific embryonic lethality) can be achieved during mass rearing. The activity of cellularization gene promoters during embryogenesis controls the timing and dose of the lethal gene product.

Results: Here, we report the isolation of the D. suzukii cellularization genes nullo, serendipity-a, bottleneck and slow-asmolasses from a laboratory strain. Conserved motifs were identified by comparing the encoded proteins with orthologs from other Drosophilids. Expression profiling confirmed that all four are zygotic genes that are strongly expressed at the early blastoderm stage. The $5^{\prime}$ flanking regions from these cellularization genes were isolated, incorporated into piggyBac vectors and compared in vitro for the promoter activities. The Dsnullo promoter showed the highest activity in the cell culture assays using D. melanogaster $\mathrm{S} 2$ cells.

Conclusions: The similarities in the gene coding and $5^{\prime}$ flanking sequence as well as in the expression pattern of the four cellularization genes between D. melanogaster and D. suzukii, suggest that conserved functions may be involved in both species. The high expression level at the early blastoderm stage of the four cellularization genes were confirmed, thus their promoters can be considered in embryonic lethality systems. While the Dsnullo promoter could be a suitable candidate, all reported promoters here are subject to further in vivo analyses before constructing potential pest control systems.
\end{abstract}

Keywords: Spotted-wing Drosophila, Sterile insect technique, Transgenic embryonic sexing system , D. melanogaster S2 cells, Tetracycline-off system

\footnotetext{
*Correspondence: ying.yan@agrar.uni-giessen.de; marc.schetelig@agrar.uni-

giessen.de

${ }^{1}$ Justus-Liebig-University Giessen, Institute for Insect Biotechnology,

Department of Insect Biotechnology in Plant Protection, Winchesterstr. 2,

35394 Giessen, Germany

Full list of author information is available at the end of the article
}

(c) The Author(s). 2020 Open Access This is an open access article distributed under the terms of the Creative Commons Attribution IGO License (https://creativecommons.org/licenses/by/3.0/igo/) which permits unrestricted use, distribution, and reproduction in any medium, provided appropriate credit to the original author(s) and the source is given. 


\section{Background}

The spotted-wing Drosophila (Drosophila suzukii Matsumura) is native to eastern Asia but has become a widespread invasive pest of fruit crops [1,2]. The sterile insect technique (SIT) is a bio-control strategy that works by releasing a large number of typically radiation-sterilized males in the environment so they can compete with wildtype males. Mating between sterilized males and wildtype females produces no offspring, so the repeated release of sterile males leads to population suppression or eradication [3-5]. SIT has been used to control tephritid fruit flies, notably the Mediterranean fruit fly (Ceratitis capitata), with great success [4]. Consequently, it is also proposed as a cost-effective and environment-friendly control strategy for D. suzukii $[6,7]$. Since sterilized females would compete with wild-type females for sterilized males and would still cause damage to fruit, $C$. capitata genetic sexing strains (GSS) have been developed to achieve maleonly release [8], which is far more efficient and costeffective than bisexual releases in the field $[9,10]$. These GSSs use recessive pupal color or temperature sensitive lethal (tsl) mutations and Y:autosome translocations thus sexing can be achieved based on the pupal color or temperature-controlled female lethality [8]. However, it is difficult to transfer genetic sexing systems to other pest species because suitable recessive mutations and chromosome rearrangements are required in the target species.

Transgenic embryonic sexing systems (TESS) have been developed in several agricultural pests to enable male-only releases by killing all females at the embryonic stage [1114]. The general mechanism involves the incorporation of binary tetracycline-off (Tet-off) modules into transposon vectors for germ-line transformation. Typically, the promoters of endogenous cellularization genes are used to express the tetracycline transactivator ( $t T A)$ gene (driver). In the absence of tetracycline, tTA binds to the tetracycline response element (TRE) and induces the TRE-linked proapoptotic gene (effector) only in females due to the presence of a sex-specific intron. Eliminating females before the feeding stage could reduce the insect diet cost during mass rearing thus lead to a more cost-effective SIT program.

A cellularization gene promoter is a key component of an efficient TESS because it controls $t T A$ expression and determines the timing and dose of effector gene expression $[13,15]$. There are four zygotic genes required for cellularization of the syncytial blastoderm in $D$. melanogaster: nullo, serendipity- $\alpha$ (sry- $\alpha)$, bottleneck (bnk) and slowas molasses (slam) [16]. A 95-bp 5' flanking sequence containing four conserved motifs is both necessary and sufficient for the blastoderm-specific expression of Dm $s r y-\alpha[17,18]$. The promoter activity at pre-blastoderm stage in $D$. melanogaster may also be controlled by TAGteam motifs, a series of 7-bp sequences commonly found in the $5^{\prime}$ flanking region of $D$. melanogaster genes expressed during early development [19]. The nullo and sry- $\alpha$ promoters have been used successfully to drive $t T A$ and induce embryonic lethality in D. melanogaster [20]. In tephritid fruit flies, the sry- $\alpha$ promoter has been used most widely, and TESS systems incorporating this promoter have been reported in Anastrepha suspensa [11], C. capitata [12] and Anastrepha ludens [21]. The bnk and nullo promoters were used in the calliphorid blowfly Lucilia cuprina [13, 14]. Thus far, the slam promoter has not been used to develop functional TESS strains, possibly due to its low activity at the embryonic stage [12, 22]. However, a TESS promoter must not be too active, if the Tet-Off system is used, because high levels of $t T A$ expression are also deleterious [23]. Therefore, systematic evaluation of cellularization genes and their promoters could facilitate the development of optimal TESS systems.

Here we identified four $D$. suzukii cellularization genes (nullo, sry- $\alpha$, bnk and slam) based on the reference genome sequence in the Spotted Wing Drosophila database, SWDbase [24]. We isolated the coding sequence (CDS) of each gene from our laboratory strain (D. suzukii USA) and confirmed the presence of conserved motifs by comparing the encoded proteins with orthologs in other Drosophila species. The expression profile of each gene during development was verified by reverse transcription (RT)-PCR and quantitative real-time (qRT) PCR. We then isolated the $5^{\prime}$ flanking sequences from the four genes and incorporated them into piggyBac vectors for in vitro evaluation. Based on the promoter activity of these genes in insect cells, we discuss their potential applications in biocontrol strategies for D. suzukii.

\section{Results \\ D. suzukii cellularization genes}

Four D. suzukii cellularization genes were identified by homology searches in SWDbase and were compared in silico to known sequences. Ds_nullo (DS10_00005287) was identified as an intron-less gene like its D. melanogaster ortholog (Dm_nullo). The full-length 642-bp Ds_ nullo CDS encodes a putative protein with 213 amino acids, most closely related to its ortholog in $D$. biarmipes (99\% similarity, 96\% identity), but also similar to its orthologs in D. melanogaster (94\%) and D. rhopaloa (92\%), with lower similarity $(57-77 \%)$ in other Drosophilds (Fig. 1a; Additional file 2 Fig. S1). The Nterminus of DsNULLO features a myristoylation site and a cluster of positively charged amino acids, which may target the protein to the plasma membrane [25]. The remainder of the polypeptide features five conserved segments (Additional file 2 Fig. S1) required for NULLO proteins to stabilize the basal junction components in the nascent cleavage furrows of blastoderm cells [26]. 


\begin{tabular}{|c|c|c|c|c|c|c|c|c|c|c|c|}
\hline $\mathbf{A}$ & \multicolumn{2}{|c|}{ DsNULLO DbNULLO } & \multicolumn{2}{|c|}{ DmNULLO DrNULLO } & \multirow{2}{*}{\begin{tabular}{|c|}
\multicolumn{1}{c}{ DserNULLO } \\
$77 \%$ \\
\end{tabular}} & \multirow{6}{*}{\begin{tabular}{|c|} 
C \\
DsBNK \\
DmBNK \\
DmirBNK \\
DkBNK \\
DoBNK
\end{tabular}} & \multirow[t]{2}{*}{ DsBNK } & \multirow{2}{*}{\begin{tabular}{|c|} 
DmBNK \\
$85 \%$
\end{tabular}} & \multirow{2}{*}{$\begin{array}{c}\text { DmirBNK } \\
70 \% \\
\end{array}$} & \multirow{2}{*}{$\begin{array}{c}\text { DkBNK } \\
71 \%\end{array}$} & \multirow{2}{*}{$\begin{array}{c}\text { DoBNK } \\
66 \%\end{array}$} \\
\hline \multirow{2}{*}{$\begin{array}{l}\text { AsNULLO } \\
\text { DbNULLO }\end{array}$} & & $99 \%$ & $94 \%$ & $92 \%$ & & & & & & & \\
\hline & $99 \%$ & & $95 \%$ & $92 \%$ & $77 \%$ & & $85 \%$ & & $70 \%$ & $73 \%$ & $67 \%$ \\
\hline DmNULLO & $94 \%$ & $95 \%$ & & $90 \%$ & $77 \%$ & & $70 \%$ & $70 \%$ & & $72 \%$ & $88 \%$ \\
\hline DrNULLO & $92 \%$ & $92 \%$ & $90 \%$ & & $76 \%$ & & $71 \%$ & $73 \%$ & $72 \%$ & & $69 \%$ \\
\hline DserNULLO & $77 \%$ & $77 \%$ & $77 \%$ & $76 \%$ & & & $66 \%$ & $67 \%$ & $88 \%$ & $69 \%$ & \\
\hline $\mathbf{B}$ & DsSRY- $\alpha$ & DmSRY- $\alpha$ & DvSRY- $\alpha$ & DhSRY- $\alpha$ & DgSRY-a & $\mathbf{D}$ & DsSLAM & DMSLAM & DpSLAM & DgSLAM & DbuSLAM \\
\hline DsSRY- $\alpha$ & & $94 \%$ & $68 \%$ & $51 \%$ & $54 \%$ & \multirow{5}{*}{$\begin{array}{l}\text { DsSLAM } \\
\text { DmSLAM } \\
\text { DpSLAM } \\
\text { DgSLAM } \\
\text { DbuSLAM }\end{array}$} & & $84 \%$ & $77 \%$ & $70 \%$ & $69 \%$ \\
\hline DmSRY- $\alpha$ & $94 \%$ & & $67 \%$ & $51 \%$ & $53 \%$ & & $84 \%$ & & $85 \%$ & $74 \%$ & $73 \%$ \\
\hline DvSRY- $\alpha$ & $68 \%$ & $67 \%$ & & $51 \%$ & $54 \%$ & & $77 \%$ & $85 \%$ & & $75 \%$ & $72 \%$ \\
\hline DhSRY- $\alpha$ & $51 \%$ & $51 \%$ & $51 \%$ & & $74 \%$ & & $70 \%$ & $74 \%$ & $75 \%$ & & $79 \%$ \\
\hline DgSRY- $\alpha$ & $54 \%$ & $53 \%$ & $54 \%$ & $74 \%$ & & & $69 \%$ & $73 \%$ & $72 \%$ & $79 \%$ & \\
\hline \multicolumn{12}{|c|}{$\begin{array}{l}\text { Fig. } 1 \text { Similarities in the NULLO, SRY-a, BNK and SLAM proteins from Drosophila spp. a Drosophila suzukii NULLO (DsNULLO) is aligned with } \\
\text { orthologs from Drosophila biarmipes [DbNULLO] (GenBank: XP_016948821.1), Drosophila melanogaster [DmNULLO] (GenBank: NP_511067.3), } \\
\text { Drosophila rhopaloa [DrNULLO] (GenBank: XP_016982709.1), and Drosophila serrata [DserNULLO] (GenBank: XP_020805612.1). b Drosophila suzukii } \\
\text { SERENDIPITY-a (DsSRY-a) is aligned with orthologs from Drosophila grimshawi [DgSRY-a] (GenBank: XP_001995347.1), Drosophila hydei [DhSRY-a] } \\
\text { (GenBank: XP_023168708.1), Drosophila melanogaster [DmSRY-a] (GenBank: NP_524580.1) and Drosophila virilis [DvSRY-a] (GenBank: } \\
\text { XP_002056142.1). c Drosophila suzukii BOTLENECK (DsBNK) is aligned with orthologs from Drosophila melanogaster [DmBNK] (GenBank: } \\
\text { NP_524604.2), Drosophila miranda [DmirBNK] (GenBank: XP_017140437.1), Drosophila obscura [DoBNK] (GenBank: XP_022213695.1), and Drosophila } \\
\text { kikkawai [DkBNK] (GenBank: XP_017034088.1).d Drosophila suzukii SLOW-AS-MOLASSES (DsSLAM) is aligned with orthologs from Drosophila } \\
\text { grimshawi [DgSLAM] (GenBank: XP_001988112.1), Drosophila pseudoobscura [DpSLAM] (GenBank: XP_001355861.2), Drosophila melanogaster } \\
\text { [DmSLAM] (GenBank: NP_001285668.1), and Drosophila busckii [DbuSLAM] (GenBank: ALC38995.1). The protein alignment was performed in } \\
\text { Geneious Prime program and similarities (Blosum45, threshold 1) are shown }\end{array}$} \\
\hline
\end{tabular}

Ds_sry- $\alpha$ (DS10_00012897) has three exons. The fulllength 1593-bp Ds_sry- $\alpha$ CDS encodes a putative protein with 530 amino acids, most closely related to its ortholog in D. melanogaster (94\% similarity, $87 \%$ identity; Fig. 1b). The N-terminus of DsSRY- $\alpha$ features a cysteine-rich motif, possibly a transmembrane segment (Additional file 2 Fig. S2). A conserved C-terminal region shows high similarity to Ezrin, Radixin and Moesin (ERM) proteins which facilitate actin-membrane interactions [27], suggesting DsSRY- $\alpha$ fulfils a similar role in the reorganization of microfilaments during cellularization [28].

Ds_bnk (DS10_00007356), like Ds_nullo, is an intronless gene. The 903-bp Ds_bnk CDS encodes a putative protein with 303 amino acids, most closely related to its ortholog in D. melanogaster (85\% similarity, 80\% identity; Fig. 1c). Finally, Ds_slam (DS10_00010822) has two exons. The 3414-bp Ds_slam CDS encodes the largest of the four cellularization proteins, with 1137 amino acids. DsSLAM is most closely related to its ortholog in D. melanogaster (88\% similarity, 74.6\% identity; Fig. 1d). Previous studies have not identified any functional motifs in DmBNK [29] or DmSLAM [30]. However, several highly conserved regions were identified in DsBNK (Additional file 2 Fig. S3) and DsSLAM (Additional file 2 Fig. S4). The phylogenetic analysis of all four proteins using a neighbor-joining algorithm revealed that DsSRY- $\alpha$, DsBNK and DsSLAM clustered with their $D$. melanogaster orthologs, whereas DsNULLO clustered with DbNULLO and DmNULLO (Fig. 2).

\section{D. suzukii cellularization genes are strongly expressed during embryogenesis}

The RT-PCR results suggest that mRNA levels of all four cellularization genes were undetectable during the $0-0.5 \mathrm{~h}$ time window, reached a sharp peak around the onset of cellularization (2-3 h after egg laying) and then declined, but nevertheless persisted to the adult stage (Additional file 2 Fig. S5). Ds_sry $\alpha$ expression peaked slightly later than Ds_nullo, as previously reported for the D. melanogaster orthologs [31]. The Ds_bnk and Ds slam transcripts were particularly abundant at the $1-3 \mathrm{~h}$ stage, but declined sharply thereafter (Additional file 2 Fig. S5). Primers for the reference genes TATA binding protein (TBP), glyceraldehyde-3-phosphate dehydrogenase $(G A P D H)$, arginine kinase $(A K)$, a-Tubulin $(\alpha-t u b)$ and Histone H3 (His3) were also evaluated for their performance using RT-PCR analysis [32, 33]. The primers that amplified products from most or all stages (Additional file 2 Fig. S5) were further evaluated for efficiency using a serial dilution of cDNA as templates. The primer 
A

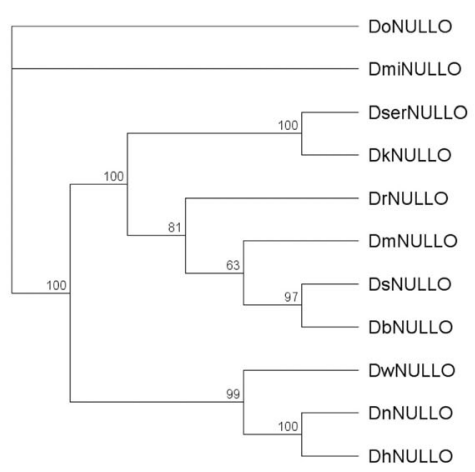

B

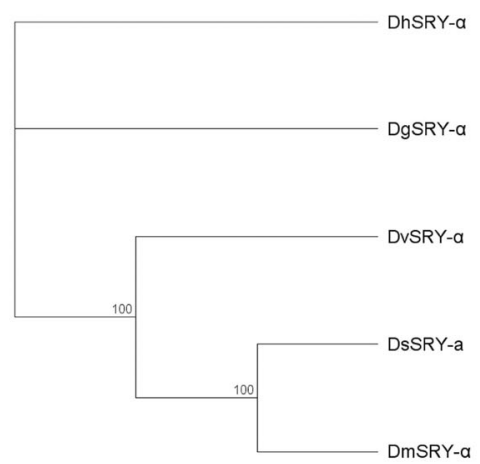

C

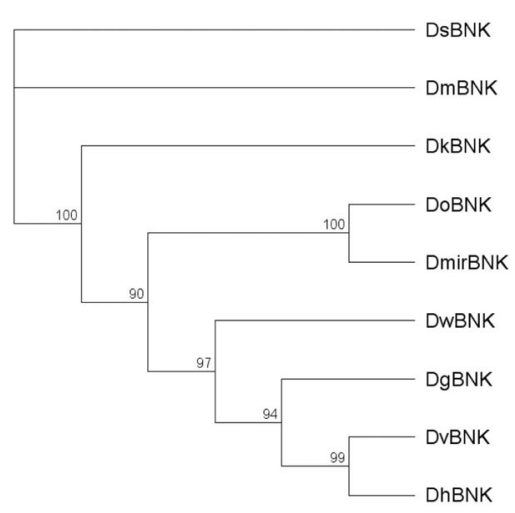

D

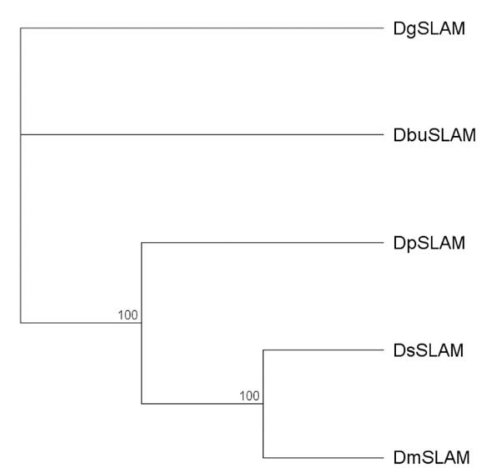

Fig. 2 Phylogenetic analysis of Drosophila spp. NULLO, SRY-a, BNK and SLAM proteins. Unrooted neighbor-joining trees were constructed with amino acid sequences from a NULLO, b SRY-a, c BNK, and $\mathbf{d}$ SLAM. Bootstrap values (1000 replicates) are shown on the nodes of the trees. In addition to the orthologs in Fig. 1a, Drosophila hydei [DhNULLO] (GenBank: XP_023161247.1), Drosophila kikkawai [DkNULLO] (GenBank: XP_017022675.1), Drosophila miranda [DmirNULLO] (GenBank: XP_017156218.1), Drosophila navojoa [DnNULLO] (GenBank: XP_017964581.1), Drosophila obscura [DoNULLO] (GenBank: XP_022210380.1), and Drosophila willistoni [DwNULLO] (GenBank: XP_002071148.1) are used for the alignment. In addition to the orthologs in Fig. 1c, Drosophila grimshawi [DgBNK] (GenBank: EDV90810.1), Drosophila hydei [DhBNK] (GenBank: XP_023168586), Drosophila virilis [DvirBNK] (GenBank: XP_002055918.1) and Drosophila willistoni [DwBNK] (GenBank: XP_002072000.1) are used for the alignment. Other species abbreviations are the same as in Fig. 1

efficiency was calculated as $99.4 \%$ for $D s_{-} T B P, 93.6 \%$ for Ds_GAPDH, $99.5 \%$ for Ds_AK, and $89.6 \%$ for Ds_His3. Consequently, primers for $D s_{-} T B P$ and $D s_{-} A K$ that showed high efficiency were used in the qRT-PCR experiments. The qRT-PCR data were consistent with our semi-quantitative RT-PCR analysis, with all four cellularization genes expressed at the highest level during the early blastoderm stages (Fig. 3). When normalized with $D s \_T B P$ and Ds_AK, the Ds_sry- $\alpha, D s \_n u l l o, D s \_b n k$ and Ds_slam transcripts were 123-, 1040-, 13,469- and 56-

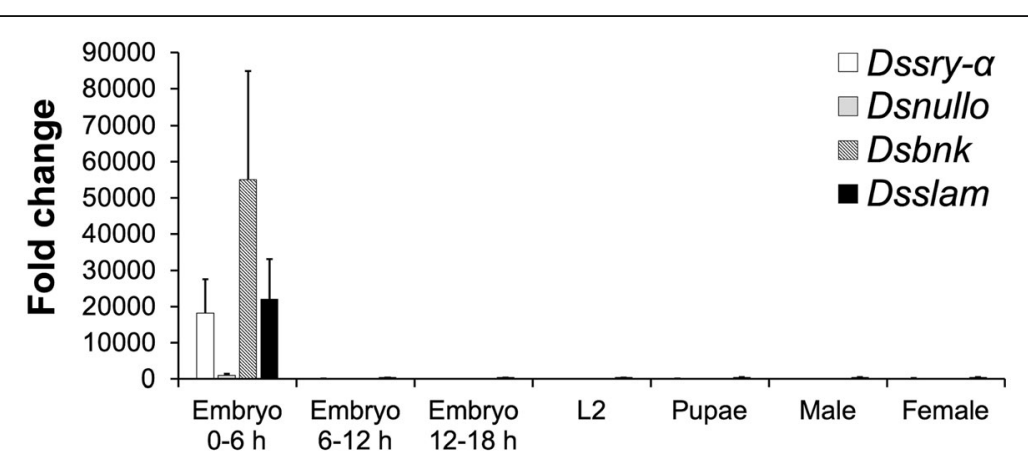

Fig. 3 Quantitative real-time-PCR to determine relative expression of cellularization genes. Three different embryonic stages $(0-6 \mathrm{~h}, 6-12 \mathrm{~h}$ and 12-18 $\mathrm{h}$ after egg laying), the second instar larvae (L2), pupae and $5 \mathrm{~d}$ old males and females were analyzed. The gene expression was normalized to the reference genes TBP and $A K$, and related to the Dsnullo expression level at 6-12 h embryos. The mean and standard error from three replicate experiments are shown 
fold more abundant in 0-6 h embryos than 6-12 h embryos, and 116-, 153-, 2433- and 49-fold more abundant in 0-6 h embryos than adult female, respectively (Fig. 3). Furthermore, in 0-6h embryos the Dssry- $\alpha$, Dsbnk and Dsslam transcripts were respectively 17.5 -fold, 52.9-fold and 21.2-fold more abundant than Dsnullo mRNA. The relatively large variations (error bars) in gene expression from 0 to $6 \mathrm{~h}$ embryos are possibly due to the different ratios of eggs collected at early blastoderm stages $(2-3 \mathrm{~h})$ to eggs from other time period within 0-6 $\mathrm{h}$ from three replicates. Nevertheless, the expression levels of all four genes were much lower at later developmental stages (Fig. 3).

\section{$5^{\prime}$ flanking region of $D$. suzukii cellularization genes}

The $5^{\prime}$ flanking region of Ds_sry- $\alpha$ lacked motif IV but contained two TAGteam motifs, as well as a TATA box (TATATAAA) $23 \mathrm{bp}$ upstream of the putative transcription start site (TSS) (Fig. 4A; Additional file 2 Fig. S6). The 5' flanking region of Ds_nullo contained one TAGteam motif and a TATA box (TATATAT) $24 \mathrm{bp}$ upstream of the predicted TSS (Fig. 4A; Additional file 2 Fig. S7). The 5' flanking region of $D s \_b n k$ contained three TAGteam motifs and a TATA box (TATATAAA) $25 \mathrm{bp}$ upstream of the TSS (Fig. 4A; Additional file 2 Fig. S8). However, neither of 5' flanking sequences of Ds_slam and Dm_slam contained
TAGteam motifs; there was also no TATA box in these regions identified (Fig. 4A; Additional file 2 Fig. S9). We prepared four piggyBac test vectors (V205-V208) in which the 5 ' flanking sequence of $D s \_s r y-\alpha, D s \_n u l l o, D s \_s l a m$ or $D s$ bnk was linked to the DsRed reporter gene, respectively (Fig. 4B). All vectors also contained a DmPUb-AmCyan marker, allowing us to use an EGFP filter to determine the efficiency of transfection. We transfected D. melanogaster S2 cells with each of the four constructs, fixed the cells $18 \mathrm{~h}$ posttransfection and counted the numbers of blue and red fluorescent cells. The ratio of red to blue cells therefore provided an internally-consistent readout of regulation activity from the $5^{\prime}$ flanking sequence. We found that the $5^{\prime}$ flanking sequence of Ds_nullo (construct V206) generated the strongest DsRed signal (Fig. 4C) and the highest DsRed:AmCyan ratio (Fig. 4D) indicating the highest activity as a gene promoter $(P=0.001$ when compared to V205 and V208, $P=$ 0.002 when compared to V207, one-way ANOVA).

\section{Discussion}

Early development in many insect species involves a syncytial blastoderm stage followed by cellularization after a certain number of nuclear divisions, and this process is orchestrated by a small number of zygotic genes [16]. Four major cellularization genes have been identified in

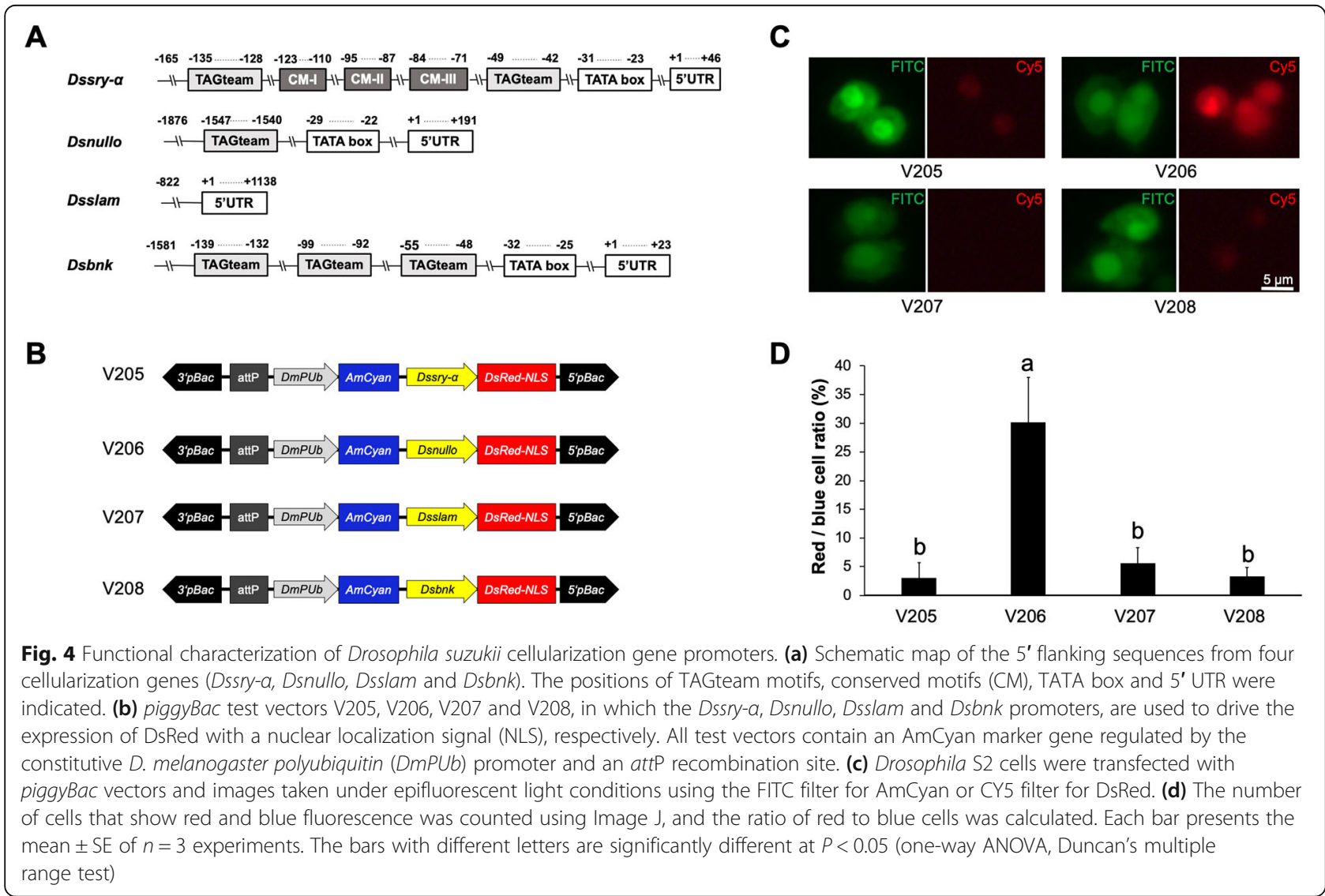


the developmental model organism D. melanogaster (nullo, sry- $\alpha$, bnk and slam), and the conserved sequences of the orthologs in D. suzukii (Additional file 2 Fig. S1-4) together with the peak of expression at the early blastoderm stage (Fig. 3) suggest that similar functions may be involved in both species, including the regulation of actin filaments, microfilaments and membrane polarization [16]. D. melanogaster embryogenesis has been divided into 17 stages, with the cellular blastoderm arising during stage $5,2 \mathrm{~h} 10 \mathrm{~min}$ and $2 \mathrm{~h} 50 \mathrm{~min}$ after egg laying [34]. During this stage, $n u l l o, s r y-\alpha, b n k$, and slam are expressed to coordinate the cellularization process, but expression declines thereafter, to low levels for nullo and bnk, and to moderate levels for sry- $\alpha$ and slam [35]. In many fly species, the expression of all four cellularization genes is typically detected as early as $1-2 \mathrm{~h}$ after egg laying [13, 22, 31]. However, the corresponding transcripts were already present in D. suzukii embryos collected $0-1 \mathrm{~h}$ after egg laying, suggesting that gene expression commences at the start of embryogenesis (Additional file 2 Fig. S5). This apparent early expression may reflect the facultative ovoviviparity of $D$. suzukii females, allowing them to retain fertilized eggs, which can therefore develop to a certain extent before laying [36]. However, the transcripts of all four cellularization genes were undetectable during the $0-0.5 \mathrm{~h}$ time window (Additional file $2 \mathrm{Fig}$. S5), confirming the absence of maternal gene expression and that egg retention was not responsible for the early transcripts - instead, the early zygotic gene expression was a genuine phenomenon.

The early and stage-specific expression is important for the development of genetic pest-control systems because it allows us to focus the effect of lethal effector transgenes at the correct developmental stage [11, 13, 37]. Specifically, constitutive and unconditional effector gene expression later in development could lead to undesired female sterility or lethality in a control strain and has been reported previously in interfering with ovarian development and reducing productivity of those strains $[14,15,21]$. Our qRT-PCR data showed that the four D. suzukii cellularization genes were expressed with a similar profile to their orthologs in D. melanogaster (Fig. 3). The considerably higher gene expression in 0-6 h embryos than 6-12 $\mathrm{h}$ embryos for Ds_nullo and Ds_bnk (1040-fold and 13,469fold, respectively), suggesting that their promoters are likely early active and would therefore be useful candidates for the development of TESS systems in D. suzukii, whereas the Ds_sry- $\alpha$ and Ds_slam promoter may allow moderate but constitutive expression at later developmental stages. Therefore, those early-embryonic promoters could be used to establish $D$. suzukii sexing systems for SIT programs in the future. Multiple promoters and independent systems will also be important in dealing with natural resistance processes [38]. Those backup systems would help establish stable lines that overcome primary- site mutations during rearing processes and quality control of the mass-reared strains, and help strains to cope with second-site maternal-effect suppressors [38].

The 5 ' flanking region of the $D$. suzukii cellularization genes contained several TAGteam motifs, which are often found in D. melanogaster genes expressed early in development, and act as binding sites for the transcriptional activator Zelda $[19,39]$. The $5^{\prime}$ flanking region of $D s \_b n k$ contains three TAGteam motifs 91-139 bp upstream of the predicted TSS, whereas that of Ds_sry- $\alpha$ contains two such motifs $93-135 \mathrm{bp}$ upstream of the predicted TSS (Fig. 4A). In contrast, the $5^{\prime}$ flanking region of Ds_nullo features only one TAGteam motif, which is much further away from the TSS: 1576 bp upstream (Fig. 4A). Such differences in the number and location of TAGteam motifs may contribute to differences in gene expression at the 0 $6 \mathrm{~h}$ embryonic stage, since $D s_{-} b n k$ and $D s \_s r y-\alpha$ mRNA were more than 10-fold more abundant than Ds_nullo mRNA (Fig. 3). Interestingly, neither the Ds_slam nor the Dm_slam 5' flanking region feature any TAGteam motifs (Fig. 4A; Additional file 2 Fig. S9) but Ds_slam was nevertheless expressed at relatively high levels during the $0-6 \mathrm{~h}$ embryonic stage. This suggests that the transcriptional activation of Ds_slam may be less dependent on Zelda compared to the other genes.

piggyBac-based vectors have been used to evaluate the embryonic activity of the promoters using the A. suspensa cell line UFENY-AsE01, an embryonic cell line derived originally from 20-h old embryos [40]. Previously, sry-a promoters from $C$. capitata and $A$. suspensa were able to mediate cell death in these AsE01 cells as well as embryonic lethality in transgenic flies [11,37, 41]. In addition, the $D$. melanogaster S2 cells are also derived from late embryonic stages [42], and have been one of the few D. melanogaster cell lines that can be used for heterologous gene expression [43]. Often high levels of heterologous protein expression in S2 cells are due to strong promoters in the expression vectors [44, 45]. Although Ds_nullo was expressed at the lowest level among the four genes at the 0-6 h embryonic stage, its promoter achieved the strongest DsRed expression in the cell culture tests (Fig. 4C, D). Such observation suggested that the Ds_nullo promoter mediated higher protein production than other tested promoters in the embryonic S2 cells. The weaker activity of $D s \_s r y-\alpha$ promoter may be due to the absence of motif IV, which was suggested to function as a positive cis-acting regulatory element. Previous reports verified that the deletion of motif IV in the Dm_sry- $\alpha$ promoter resulted in a more than four-fold loss of activity at the blastoderm stage [18]. Weak transgene expression was also observed for the slam promoters from C. capitata [12] and L. cuprina [22], which do not have TATA box motifs. Similarly, both $D m_{-}$ slam and Ds_slam promoter sequences lack TATA box motifs (Additional file 2 Fig. S9). Such TATA-less genes 
often show multiple TSSs [46], and are more flexibly regulated compared to TATA-containing genes [47]. Thus, slam in these species may involve multiple elements for its correct regulation. Computational and functional studies in other species revealed an importance for transcriptional activity based on motifs in the core promoter sequence [48] and a number of relevant transcription factors involved have been studied [47]. But ultimately, the reported promoters have to be functionally tested to evaluate their potential for pest control applications.

\section{Conclusions}

For D. melanogaster, the gene networks that regulate early development were intensively studied to understand the basic biological processes such as cellularization, sex determination and patterning $[16,19,39,48]$. Here we have verified the similarities and differences in the promoter and CDS sequences of four cellularization genes between D. melanogaster and D. suzukii. More functional studies are needed at transcription or protein level [17, 29-31] to understand the gene interaction and network connection during early development in $D$. suzukii. In addition, $D$. melanogaster S2 cells are potentially a good system to study early genes and their promoters due to its embryonic origin [42]. Our results indicated that the Ds_nullo promoter can be considered for driving embryonic lethality in a genetic control system such as a TESS. Nevertheless, all reported promoters here have to be further functionally analysed to evaluate their in vivo performance before constructing potential pest control systems.

\section{Methods}

\section{Insect rearing and sample collection}

Wild-type $D$. suzukii USA specimens were maintained at $25^{\circ} \mathrm{C}$ and $60 \%$ humidity with a 12 -h photoperiod. Eggs were collected over a 30-min or 1-h period on grape juice agar plates as previously described [49]. We allowed the eggs to develop to the desired developmental time point before freezing them in liquid nitrogen. The larvae and pupae were directly collected from stock vials at the desired stage. Adult males and females were isolated immediately after eclosion and sampled 1 or 5 days later.

\section{Gene sequence isolation and analysis}

The gene query function was not available in SWDbase [24] when this work was initiated in 2014. Therefore, the CDS of the D. melanogaster genes Dm_sry- $\alpha$ (FBgn0003510), Dm_nullo (FBgn0004143), Dm_bnk (FBgn0004389) and Dm_slam (FBgn0043854) were obtained from FlyBase (http://flybase.org/) and were used as tBLASTx queries against SWDbase. The Ds_sry- $\alpha$ query matched a 1593-bp CDS on scaffold 705. Ds nullo matched a 642-bp CDS on scaffold 5. Ds_bnk matched a 903-bp CDS on scaffold 21. Finally, Ds_slam matched a 1677-bp CDS on scaffold 181 .
Based on these hits, primers were designed to amplify the full-length CDS of each gene from cDNA prepared from wild-type D. suzukii USA embryos (Additional File 1). Total RNA was isolated from embryos at the early blastoderm stage (3-4h after egg laying) using the ZR Tissue \& Insect RNA MicroPrep kit (Zymo Research) and residual DNA was removed with Turbo DNAse (Ambion). The iScript cDNA Synthesis Kit (Bio-Rad) was used to prepare cDNA from $1 \mu \mathrm{g}$ DNA-free RNA. For Ds_bnk, Ds_slam, and Ds_ nullo, each $25-\mu \mathrm{l}$ reaction mix comprised $0.2 \mu \mathrm{L}$ Platinum Taq DNA polymerase (Invitrogen), $2.5 \mu \mathrm{L}$ 10x PCR buffer, $0.75 \mu \mathrm{L} 50 \mathrm{mM} \mathrm{MgCl} 2,1.0 \mu \mathrm{L} 10 \mathrm{mM}$ dNTP mix, $1.0 \mu \mathrm{L}$ of each primer $(0.4 \mu \mathrm{M})$, and $0.5 \mu \mathrm{L} \mathrm{cDNA}$. An initial denaturation step at $95^{\circ} \mathrm{C}$ for $2 \mathrm{~min}$ was followed by 35 cycles of denaturation at $95^{\circ} \mathrm{C}$ for $30 \mathrm{~s}$, annealing at $52^{\circ} \mathrm{C}$ for $30 \mathrm{~s}$ and extension at $72^{\circ} \mathrm{C}$ for $1 \mathrm{~min}$ (nullo and bnk) or $2 \mathrm{~min}$ (slam), and a final extension at $72{ }^{\circ} \mathrm{C}$ for $5 \mathrm{~min}$. For Ds_sry$\alpha$, multiple PCR attempts using the Platinum Taq DNA polymerase did not result in amplificates, thus the Phusion Flash High-Fidelity DNA Polymerase (Thermo Fisher Scientific) was used. PCR was carried out using $10 \mu \mathrm{L}$ of the Phusion Flash High-Fidelity PCR Master mix with $1.0 \mu \mathrm{L}$ of each primer and $1.0 \mu \mathrm{L}$ cDNA $(0.4 \mu \mathrm{M})$ in a $25-\mu$ reaction. An initial denaturation step at $98^{\circ} \mathrm{C}$ for $10 \mathrm{~s}$ was followed by 30 cycles of denaturation at $98^{\circ} \mathrm{C}$ for $30 \mathrm{~s}$, annealing at $52^{\circ} \mathrm{C}$ for $5 \mathrm{~s}$ and extension at $72{ }^{\circ} \mathrm{C}$ for $30 \mathrm{~s}$, a final extension at $72{ }^{\circ} \mathrm{C}$ for $1 \mathrm{~min}$, and a $4{ }^{\circ} \mathrm{C}$ hold. The PCR products were separated by $1 \%$ agarose gel electrophoresis and extracted from the gels using the QIAquick Gel Extraction Kit (Qiagen) before cloning in vector pCR4-TOPO. The presence of inserts was confirmed by restriction digestion with EcoRI and sequencing using M13 primers. Sequence translation, polypeptide alignment and phylogenetic analysis were performed using the Geneious Prime software [50].

\section{Gene expression analysis by RT-PCR and qRT-PCR}

The RT-PCR was assembled using platinum Taq polymerase as described above. The reaction profile comprised an initial denaturation step at $94{ }^{\circ} \mathrm{C}$ for $4 \mathrm{~min}$, followed by 35 cycles of denaturation at $94{ }^{\circ} \mathrm{C}$ for $30 \mathrm{~s}$, annealing at $55^{\circ} \mathrm{C}$ (His3, TBP, nullo, bnk and slam) or $60^{\circ} \mathrm{C}(A K$, GADPH, $\alpha-T u b$, sry- $\alpha$ ) for $30 \mathrm{~s}$, and extension at $72{ }^{\circ} \mathrm{C}$ for $1 \mathrm{~min}$, and a final extension at $72{ }^{\circ} \mathrm{C}$ for 4 min. We used SsoAdvanced Universal SYBR Green Supermix (Bio-Rad) with $100 \mathrm{ng}$ of template cDNA for the qRT-PCR experiments. The reactions were performed in a CFX96 Touch Real-Time PCR Detection System (Bio-Rad) and each comprised an initial denaturation step at $95^{\circ} \mathrm{C}$ for $30 \mathrm{~s}$, followed by 40 cycles of denaturation at $95^{\circ} \mathrm{C}$ for $10 \mathrm{~s}$, annealing at $60^{\circ} \mathrm{C}$ for $20 \mathrm{~s}$, extension at $65^{\circ} \mathrm{C}$ for $5 \mathrm{~s}$, and a machine related $95^{\circ} \mathrm{C}$ for $0.5 \mathrm{~s}$ step. All experiments were carried out in three biological triplicates each with three technical replicates. 
The $2^{-\Delta \Delta C t}$ formula was used for all samples and values were normalized to the geometric mean of the reference genes $T B P$ and $A K$. All primer sequences were listed in Additional file 1.

\section{Promoter isolation and plasmid construction}

The upstream flanking sequences of the $D$. melanogaster and D. suzukii cellularization genes were obtained from FlyBase and SWDbase, respectively. The sequences were aligned using Geneious and searched for conserved motifs, TAGteam motifs [19] and the TATA box. Highmolecular-weight genomic DNA was prepared from $D$. suzukii adults using DNAzol Reagent (Thermo Fisher Scientific). Promoter fragments were amplified from genomic DNA using the primers listed in Additional file 1 and were transferred to pCR4-TOPO as described above. After confirming the nucleotide sequences, the promoter fragments were amplified and used to replace the D. melanogaster polyubiquitin (DmPUb) promoter in the piggyBac vector pXLBacII-attP-PUbAmCyan_DmPUb_DsRed-NLS-SV40

[51] at the Bsu36I and MluI restriction sites, to obtain the test vectors V205-V208.

\section{Cell culture experiments}

Drosophila Schneider 2 (S2) cells [42] were grown on Schneider's medium containing 10\% heat-inactivated fetal bovine serum (Hi-FBS) and 1\% penicillin/streptomycin in closed-capped flasks without $\mathrm{CO}_{2}$ at $25^{\circ} \mathrm{C}$. Cells were passaged every 2 days until $\geq 90 \%$ viability was achieved. For transient transfection, we used Xfectin transfection reagent (Takara) according to the manufacturer's instructions. We placed a 13-mm TC coverslip (Sarstedt) into each well of a 24-well plate to facilitate imaging, then seeded each well with $5 \times 10^{5}$ cells in $500 \mu \mathrm{L}$ of medium. After $3 \mathrm{~h}$, the settled cells were transfected using $2 \mu \mathrm{g}$ of plasmid DNA, 0.6 $\mu \mathrm{L}$ Xfectin, 27.4 $\mu \mathrm{L}$ Xfectin buffer and $270 \mu \mathrm{L}$ serum-free Schneider's medium for $4 \mathrm{~h}$. Transfection was stopped by refreshing the dishes with $500 \mu \mathrm{L}$ Schneider's medium containing $10 \% \mathrm{Hi}-\mathrm{FBS}$ and $1 \%$ penicillin/streptomycin. The cells were incubated for $\sim 18 \mathrm{~h}$ at $25^{\circ} \mathrm{C}$ before fixing in $4 \%$ paraformaldehyde for $15 \mathrm{~min}$ and washing briefly with PBS prior to microscopy.

\section{Cell imaging and counting}

Drosophila S2 cells transfected with constructs V205V208 were imaged using an inverted microscope equipped with a fluorescent slide containing the FITC (ex: 490/20; detecting also AmCyan expression) and CY5 (ex: 620/60) filters (Leica DM IL LED, Leica Microsystems, Wetzlar, Germany). All settings (e.g., exposure time, gain, magnification) were identical for each test so that the fluorescence intensity could be compared. Fluorescent cells were counted in Image J (Fiji) using the automated cell count function. Specifically, raw images were converted to an 8- bit standardized format and inverted before a watershed was applied to separate any cells in direct contact. The counting threshold was set to 30 for the DsRed filter. The differences in red:blue cell ratios (data were square root transformed) for different piggyBac vectors were tested by one-way analysis of variance (ANOVA) and means were separated using Duncan's multiple range test in SigmaPlot v12.5 (Systat Software).

\section{Supplementary Information}

The online version contains supplementary material available at https://doi. org/10.1186/s12863-020-00939-y.

\section{Additional file 1. Primer sequences.}

Additional file 2: Figure S1. Drosophila suzukii NULLO protein alignment. DsNULLO is aligned with orthologs from Drosophila biarmipes [DbNULLO] (GenBank: XP_016948821.1), Drosophila hydei [DhNULLO] (GenBank: XP_023161247.1), Drosophila kikkawai [DkNULLO] (GenBank: XP_017022675.1), Drosophila melanogaster [DmNULLO] (GenBank: NP_511067.3), Drosophila miranda [DmirNULLO] (GenBank: XP_017156218.1), Drosophila navojoa [DnNULLO] (GenBank: XP_017964581.1), Drosophila obscura [DoNULLO] (GenBank: XP_022210380.1), Drosophila rhopaloa [DrNULLO] (GenBank: XP 016982709.1), Drosophila serrata [DserNULLO] (GenBank: XP_020805612.1) and Drosophila willistoni [DwNULLO] (GenBank: XP_002071148.1). Identical amino acids are shaded black and conservative changes are indicated in gray. All proteins contain a consensus site for N-terminal myristoylation (M) followed by a positively charged cluster $(P)$. The remainder of the protein contains five conserved regions of amino acids (A-E) separated by short non-conserved regions. Figure S2. Drosophila suzukii SERENDIPITY-a (SRY-a) protein alignment. DsSRY-a is aligned with orthologs from Drosophila grimshawi [DgSRY-a] (GenBank: XP_001995347.1), Drosophila hydei [DhSRY-a] (GenBank: XP_023168708.1), Drosophila melanogaster [DmSRY-a] (GenBank: NP_524580.1) and Drosophila virilis [DvSRY-a] (GenBank: XP_002056142.1). The putative transmembrane domain is underlined and the region of similarity with proteins of the ERM family is boxed. Figure S3. Drosophila suzukii BOTTLENECK (BNK) protein alignment. DsBNK is aligned with orthologs from Drosophila grimshawi [DgBNK] (GenBank: EDV90810.1), Drosophila hydei [DhBNK] (GenBank: XP_023168586), Drosophila kikkawai [DkBNK] (GenBank: XP_017034088.1), Drosophila melanogaster [DmBNK] (GenBank: NP_524604.2), Drosophila miranda [DmirBNK] (GenBank: XP_017140437.1), Drosophila obscura [DoBNK] (GenBank: XP_022213695.1), Drosophila virilis [DvirBNK] (GenBank: XP_002055918.1) and Drosophila willistoni [DwBNK] (GenBank: XP_002072000.1). Highly conserved regions are marked with red lines. Figure S4. Drosophila suzukii SLOW-AS-MOLASSES (SLAM) protein alignment. DsSLAM is aligned with orthologs from Drosophila grimshawi [DgSLAM] (GenBank: XP_001988112.1), Drosophila pseudoobscura [DpSLAM] (GenBank: XP_001355861.2), Drosophila melanogaster [DmSLAM] (GenBank: NP_001285668.1), and Drosophila busckii [DbuSLAM] (GenBank: ALC38995.1). Highly conserved regions are marked with red lines. Figure S5. Reverse-transcriptase (RT)-PCR to evaluate the reference (a) and cellularization (b) genes through the development of Drosophila suzukii. Primer sequences for reference genes TATA binding protein (TBP), glyceraldehyde-3-phosphate dehydrogenase (GAPDH), arginine kinase (AK), a-Tubulin (a-Tub) and Histone H3 (His3) (Zhai et al., 2014; Li and Handler, 2017), as well as for four cellularization genes can be found in "Additional file 1". Embryos collected at different time points after egg laying (in hours), larvae (first, second and third instar), pupae (2 days after prepupae), adult female and male ( 1 and 5 days old). Additional 0-0.5 h samples were used for cellularization genes. The PCR product sizes are 140 bp for AK, 130 bp for GAPDH, 189 bp for $a-T u b, 129$ bp for His3, and 182 bp for TBP, $161 \mathrm{bp}$ for sry-a, $155 \mathrm{bp}$ for nullo, $159 \mathrm{bp}$ for bnk, and $149 \mathrm{bp}$ for slam. $M$ is the molecular weight ladder. Figure S6. Alignment of the Ds_sry- $a$ and Dm_sry- $a$ 5' flanking sequences. The upstream flanking sequence from Ds_sry-a (165 bp, before it reaches the upstream gene 
DS10_00012896, the ortholog of D. melanogaster janus A) and Dm_sry-a (167 bp) together with the $5^{\prime}$-UTR (annotated in green) are compared. The $5^{\prime}$ flanking sequences of Ds sry-a and Dm_sry-a contain three and four conserved motifs that confer blastoderm-specific expression, respectively. Both $5^{\prime}$ flanking sequences contain two TAGteam motifs (annotated in yellow), and a TATA box (TATATAAA) 23 bp upstream of the putative transcription start site. The Ds_sry- $a 5^{\prime}$ flanking sequence was fused to DsRed-NLS in V205 to act as a gene promoter for the in vitro test. Figure S7. Alignment of the Ds_nullo and Dm_nullo 5' flanking sequences. The upstream flanking sequence from Ds_nullo (1876 bp) and Dm_nullo $(1753 \mathrm{bp})$ together with the $5^{\prime}$-UTR (annotated in green) are compared. The $5^{\prime}$ flanking sequences of Ds_nullo sequence contains one TAGteam motif (annotated in yellow), whereas that of Dm_nullo contains none. The TATA box (TATATAT) is 24 bp upstream of the putative transcription start site. The Ds_nullo 5' flanking sequence was fused to DsRed-NLS in V206 to act as a gene promoter for the in vitro test. Figure S8. Alignment of the Ds_bnk and Dm_bnk $5^{\prime}$ flanking sequences. The upstream flanking sequence from Ds_bnk (1581 bp) and Dm_bnk (1523 bp) together with the 5' UTR (annotated in green) are compared. The $5^{\prime}$ flanking sequences of Ds_bnk and Dm_bnk contain three and four TAGteam motifs (annotated in yellow), respectively, and the TATA box (TATATAAA) is $25 \mathrm{bp}$ upstream of the putative transcription start site. The Ds_bnk 5' flanking sequence was fused to DsRed-NLS in V208 to act as a gene promoter for the in vitro test. Figure S9. Alignment of the Ds_slam and Dm_slam 5' flanking sequences. The upstream flanking sequence from Ds_slam (822 bp) and Dm_slam (887 bp) together with the 5' UTRs (annotated in green) are compared. Neither the Ds_slam nor Dm_slam $5^{\prime}$ flanking sequences contain TAGteam motifs or a TATA box. The Ds_slam 5' flanking sequence was fused to DsRed-NLS in V207 to act as a gene promoter for the in vitro test.

\section{Acknowledgements}

We thank Bashir Hosseini for assistance with the cell culture experiments. This study has benefitted from discussions at the International Atomic Energy Agency funded meetings for the Coordinated Research Project 'Comparing Rearing Efficiency and Competitiveness of Sterile Male Strains Produced by Genetic, Transgenic or Symbiont-based Technologies'.

\section{About this supplement}

This article has been published as part of BMC Genetics Volume 21 Supplement 2, 2020: Comparing rearing efficiency and competitiveness of sterile male strains produced by genetic, transgenic or symbiont-based technologies. The full contents of the supplement are available online at https://bmcgenet. biomedcentral.com/articles/supplements/volume-21-supplement-2.

\section{Authors' contributions}

Y.Y., S.A.J., J.S., and C.S. performed the research. Y.Y. and M.F.S. conceived the study, analyzed data and wrote the manuscript. All authors read and approved the final manuscript

\section{Funding}

Funding provided by the Emmy Noether program of the German Research Foundation (SCHE 1833/1-1; to MFS) and the Fraunhofer Attract program of the Fraunhofer Society ('Applications for population control of D. suzukii'; to MFS). These funding bodies played no role in the design of the study and collection, analysis, and interpretation of data and in writing the manuscript. Publication costs are funded by the Joint FAO/IAEA Division of Nuclear Techniques in Food and Agriculture, IAEA (CRP No.: D4.20.16) Vienna, Austria.

\section{Availability of data and materials}

All data generated or analyzed during this study are included in this published article [and its supplementary information files]. The GenBank accession numbers are as follows: Ds_sry-a mRNA: MK392555; Ds_nullo mRNA: MK392556; Ds_bnk mRNA: MK392557; Ds_slam mRNA: MK392558.

\section{Ethics approval and consent to participate}

Not applicable.

\section{Consent for publication}

Not applicable.

\section{Competing interests}

The authors declare that they have no competing interests.

\section{Author details}

'Justus-Liebig-University Giessen, Institute for Insect Biotechnology, Department of Insect Biotechnology in Plant Protection, Winchesterstr. 2, 35394 Giessen, Germany. ${ }^{2}$ Fraunhofer Institute for Molecular Biology and Applied Ecology IME, 35394 Giessen, Germany.

Published: 18 December 2020

\section{References}

1. Walsh DB, Bolda MP, Goodhue RE, Dreves AJ, Lee J, Bruck DJ, Walton VM, O'Neal SD, Zalom FG. Drosophila suzukii (Diptera: Drosophilidae): invasive pest of ripening soft fruit expanding its geographic range and damage potential. J Integr Pest Manag. 2011;2(1):1-7.

2. Cini A, loriatti C, Anforna G. A review of the invasion of Drosophila suzukii in Europe and a draft research agenda for integrated pest management. Bull Insectol. 2012;65(1):149-60.

3. Knipling EF. Possibilities of insect control or eradication through the use of sexually sterile males. J Econ Entomol. 1955;48:459-62.

4. Klassen W, Curtis CF. History of the sterile insect technique. Dordrecht: Springer; 2005.

5. Scott MJ, Concha C, Welch JB, Phillips PL, Skoda SR. Research Advances in the Screwworm Eradication Program Over the Past 25 Years. Entomol Exp Appl. 2017;164:226-36.

6. Nikolouli K, Colinet H, Renault D, Enriquez T, Mouton L, Gibert P, Sassu F, Cáceres C, Stauffer C, Pereira R, et al. Sterile insect technique and Wolbachia symbiosis as potential tools for the control of the invasive species Drosophila suzukii. J Pest Sci. 2018;91:489-503.

7. Schetelig MF, Lee KZ, Otto S, Talmann L, Stokl J, Degenkolb T, Vilcinskas A, Halitschke R. Environmentally sustainable pest control options for Drosophila suzukii. J Appl Entomol. 2018;142(1-2):3-17.

8. Franz G. Genetic sexing strains in Mediterranean fruit Fly, an example for other species amenable to large-scale rearing for the sterile insect technique. Dordrecht: Springer; 2005

9. Caceres C, Cayol JP, Enkerlin W. Comparison of Mediterranean fruit fly (Ceratitis capitata)(Tephritidae) bisexual and genetic sexing strains: development, valuation and economics. In: Proceedings of 6th International Fruit Fly Symposium. Vienna: IAEA; 2002. p. 367-81.

10. Rendon P, McInnis D, Lance D, Stewart J. Medfly (Diptera: Tephritidae) genetic sexing: large-scale field comparison of males-only and bisexual sterile fly releases in Guatemala. J Econ Entomol. 2004;97:1547-53.

11. Schetelig MF, Handler AM. A transgenic embryonic sexing system for Anastrepha suspensa (Diptera: Tephritidae). Insect Biochem Mol Biol. 2012;42: 790-5.

12. Ogaugwu CE, Schetelig MF, Wimmer EA. Transgenic sexing system for Ceratitis capitata (Diptera: Tephritidae) based on female-specific embryonic lethality. Insect Biochem Mol Biol. 2013;43:1-8.

13. Yan Y, Scott MJ. A transgenic embryonic sexing system for the Australian sheep blow fly Lucilia cuprina. Sci Rep. 2015;5:16090.

14. Yan Y, Williamson ME, Davis RJ, Andere AA, Picard CJ, Scott MJ. Improved transgenic sexing strains for genetic control of the Australian sheep blow fly Lucilia cuprina using embryo-specific gene promoters. Mol Gen Genomics. 2020;295:287-98.

15. Yan Y, Linger RJ, Scott MJ. Building early-larval sexing systems for genetic control of the Australian sheep blow fly Lucilia cuprina using two constitutive promoters. Sci Rep. 2017;7:2538.

16. Mazumdar A, Mazumdar M. How one becomes many: blastoderm cellularization in Drosophila melanogaster. BioEssays. 2002;24(11):1012-22.

17. Ibnsouda S, Schweisguth F, de Billy G, Vincent A. Relationship between expression of serendipity- $a$ and cellularization of the Drosophila embryo as revealed by intraspecific transformation. Development. 1993;119:471-83.

18. Ibnsouda S, Schweisguth F, Jullien D, Kucherer C, Lepesant JA, Vincent A. Evolutionarily conserved positive and negative cis-acting elements control the blastoderm-specific expression of the Drosophila serendipity a cellularization gene. Mech Dev. 1995:49:71-82.

19. ten Bosch JR, Benavides JA, Cline TW. The TAGteam DNA motif controls the timing of Drosophila pre-blastoderm transcription. Development. 2006;133: 1967-77. 
20. Horn C, Wimmer EA. A transgene-based, embryo-specific lethality system for insect pest management. Nat Biotechnol. 2003;21(1):64-70.

21. Schetelig MF, Targovska A, Meza JS, Bourtzis K, Handler AM. Tetracyclinesuppressible female lethality and sterility in the Mexican fruit fly, Anastrepha ludens. Insect Mol Biol. 2016;25:500-8.

22. Edman RM, Linger RJ, Belikoff EJ, Li F, Sze SH, Tarone AM, Scott MJ. Functional characterization of calliphorid cell death genes and cellularization gene promoters for controlling gene expression and cell viability in early embryos. Insect Mol Biol. 2015;24:58-70.

23. Fu G, Condon KC, Epton MJ, Gong P, Jin L, Condon GC, Morrison NI, Dafa'alla TH, Alphey L. Female-specific insect lethality engineered using alternative splicing. Nat Biotechnol. 2007;25:353e357.

24. Chiu JC, Jiang X, Zhao L, Hamm CA, Cridland JM, Saelao P, Hamby KA, Lee EK, Kwok RS, Zhang G, et al. Genome of Drosophila suzukii, the spotted wing drosophila. G3. 2013;3(12):2257-71.

25. Resh MD. Fatty acylation of proteins: new insights into membrane targeting of myristoylated and palmitoylated proteins. Biochim Biophys Acta. 1999; 1451:1-16.

26. Hunter C, Sung P, Schejter ED, Wieschaus E. Conserved domains of the nullo protein required for cell-surface localization and formation of adherens junctions. Mol Biol Cell. 2002;13:146-57.

27. Tsukita S, Yonemura S, Tsukita S. ERM proteins: head-to- tail regulation of actin-plasma membrane interaction. Trends Biochem Sci. 1997;22:53-8.

28. Ibnsouda S, Ferrer P, Vincent A. Conservation of read-through transcription of the Drosophila serendipity genes during evolution is gratuitous. Mol Gen Genet. 1998;259(5):484-90.

29. Schejter ED, Wieschaus E. Bottleneck acts as a regulator of the microfilament network governing cellularization of the Drosophila embryo. Cell. 1993;75:373-85.

30. Lecuit T, Samanta R, Wieschaus E. Slam encodes a developmental regulator of polarized membrane growth during cleavage of the Drosophila embryo. Dev Cell. 2002;2:425-36.

31. Rose LS, Wieschaus E. The Drosophila cellularization gene nullo produces a blastoderm-specific transcript whose levels respond to the nucleocytoplasmic ratio. Genes Dev. 1992;6:1255-68.

32. Zhai YF, Lin QC, Zhou XH, Zhang XY, Liu TL, Yu Y. Identification and validation of reference genes for quantitative real-time PCR in Drosophila suzukii (Diptera: Drosophilidae). PLoS One. 2014:9:e106800.

33. Li J, Handler AM. Temperature-dependent sex-reversal by a transformer-2 gene-edited mutation in the spotted wing drosophila, Drosophila suzukii. Scientific Rep. 2017;7(1):12363.

34. Campos-Ortega JA, Hartenstein V. The embryonic development of Drosophila melanogaster, vol. Second. Berlin: Springer-Verlag; 1997.

35. Graveley BR, Brooks AN, Carlson JW, Duff MO, Landolin JM, Yang L, Artieri CG, van Baren MJ, Boley N, Booth BW, et al. The developmental transcriptome of Drosophila melanogaster. Nature. 2011;471(7339):473-9.

36. Wang XG, Stewart TJ, Biondi A, Chavez BA, Ingels C, Caprile J, Grant JA, Walton VM, Daane KM. Population dynamics and ecology of Drosophila suzukii in Central California. J Pest Sci. 2016;89:701-12.

37. Schetelig MF, Caceres C, Zacharopoulou A, Franz G, Wimmer EA. Conditional embryonic lethality to improve the sterile insect technique in Ceratitis capitata (Diptera: Tephritidae). BMC Bio. 2009;7:4.

38. Zhao Y, Schetelig MF, Handler AM. Genetic breakdown of a Tet-off conditional lethality system for insect population control. Nat Commun. 2020:11(1):3095

39. Nien CY, Liang HL, Butcher S, Sun Y, Fu S, Gocha T, Kirov N, Manak JR, Rushlow C. Temporal coordination of gene networks by Zelda in the early Drosophila embryo. PLoS Genet. 2011;7:e1002339.

40. Shi $X Z$, Lawrence PO. An embryonic cell line from the Caribbean fruit fly, Anastrepha suspensa (Diptera : Tephritidae). In Vitro Cell Dev-An. 1999;35(1): $12-4$.

41. Schetelig MF, Handler AM. Strategy for enhanced transgenic strain development for embryonic conditional lethality in Anastrepha suspensa. Proc Natl Acad Sci, USA. 2012;109(24):9348-53.

42. Schneider I. Cell lines derived from late embryonic stages of Drosophila melanogaster. J Embryol Exp Morphol. 1972;27(2):353-65.

43. Cherbas $L$, Moss R, Cherbas P. Transformation techniques for Drosophila cell-lines. Method Cell Biol. 1994;44:161-79.

44. Lemos MAN, dos Santos AS, Astray RM, Pereira CA, Jorge SAC. Rabies virus glycoprotein expression in Drosophila S2 cells. I: design of expression/ selection vectors, subpopulations selection and influence of sodium butyrate and culture medium on protein expression. J Biotechnol. 2009; 143(2):103-10.

45. Ventini DC, Astray RM, Lemos MAN, Jorge SAC, Riquelme CC, Suazo CAT, Tonso A, Pereira CA. Recombinant rabies virus glycoprotein synthesis in bioreactor by transfected Drosophila melanogaster \$2 cells carrying a constitutive or an inducible promoter. J Biotechnol. 2010;146(4):169-72.

46. Anish R, Hossain MB, Jacobson RH, Takada S. Characterization of transcription from TATA-Less promoters: identification of a new core promoter element XCPE2 and analysis of factor requirements. PLoS One. 2009;4(4):e5103.

47. Bae $\mathrm{SH}, \mathrm{Han} \mathrm{HW}$, Moon J. Functional analysis of the molecular Interactions of TATA box-containing genes and essential genes. PLoS One. 2015;10(3): e0120848.

48. Ohler U, Liao GC, Niemann H, Rubin GM. Computational analysis of core promoters in the Drosophila genome. Genome Biol. 2002;3(12):RESEARCH 0087.

49. Schetelig MF, Handler AM. Germline transformation of the spotted wing drosophilid, Drosophila suzukii, with a piggyBac transposon vector. Genetica. 2013;141(4-6):189-93.

50. Kearse M, Moir R, Wilson A, Stones-Havas S, Cheung M, Sturrock S, Buxton S, Cooper A, Markowitz S, Duran C, et al. Geneious basic: an integrated and extendable desktop software platform for the organization and analysis of sequence data. Bioinformatics. 2012;28:1647-9.

51. Schwirz J, Yan Y, Franta Z, Schetelig MF. Bicistronic expression and differential localization of proteins in insect cells and Drosophila suzukii using picornaviral 2A peptides. Insect Biochem Mol Biol. 2020;119:103324.

\section{Publisher's Note}

Springer Nature remains neutral with regard to jurisdictional claims in published maps and institutional affiliations.

Ready to submit your research? Choose BMC and benefit from:

- fast, convenient online submission

- thorough peer review by experienced researchers in your field

- rapid publication on acceptance

- support for research data, including large and complex data types

- gold Open Access which fosters wider collaboration and increased citations

- maximum visibility for your research: over $100 \mathrm{M}$ website views per year

At $\mathrm{BMC}$, research is always in progress.

Learn more biomedcentral.com/submissions 